\title{
Crecimiento y mortalidad de la concha nácar Pteria sterna en bancos silvestres de Baja California Sur, México
}

\author{
Humberto Wright-López ${ }^{1}$, Oscar Holguín-Quiñones ${ }^{1}$, Francisco Arreguín-Sánchez ${ }^{1} \&$ \\ Irene Roque-Villada ${ }^{1}$ \\ 1. Centro Interdisciplinario de Ciencias Marinas-IPN. Playa el Conchalito S/N. Apdo. Postal 592, C. P. 23000. La Paz, \\ Baja California Sur, México; marlin9119@yahoo.com.mx, oholguin@ipn.mx, farregui@ipn.mx, \\ ivillada@yahoo.com
}

Recibido 15-II-2008. Corregido 06-VIII-2008. Aceptado 08-IX-2008.

\begin{abstract}
Growht and mortality of the mollusk Pteria sterna in wild banks of Southern Baja California, Mexico. The Gulf of California nacre shell Pteria sterna is an important marine resource in Mexico, and the main species for "half" and round pearl production in the American continent. Wild spat can only be extracted legally for research or culture activities. This study calculates growth and mortality parameters for wild stocks in the eastern coast of Southern Baja California. Height's data (353 dorso-ventral measurements) were taken from 1997 to 1999 at Santa Rosalía, Mulegé and Loreto Bay coast. The maximum length was estimated at $130.35 \mathrm{~mm}$ (124.22-136.48 mm, $\mathrm{p}>0.95)$. The length frequency data were set in a "virtual year". Seasonal von Bertalanffy growth parameters were calculated in the ELEFAN I routine, the values are: $L_{\infty}=156.02 \mathrm{~mm}, k=0.48$ year $^{-1}$, $t_{0}=-0.216$ years, $\mathrm{C}=0.43, \mathrm{WP}=0.73$. and $t_{s}=0.5$. The growth performance index was $\Phi^{\prime}=4.068$. The total mortality was calculated from length-converted catch curve $Z=3.34$ year $^{-1}$. The length-weight relationship was fixed for the equation $\mathrm{W}=0.000756 * \mathrm{~L}_{(\mathrm{i})} 2.7075\left(\mathrm{r}^{2}=0.90, \mathrm{n}=233\right)$. The growth parameters values in experimental repopulation conditions are quite different, in contrast with the growth estimated in wild stocks oysters. The value of total mortality is similar to the estimations for Pinctada fucata populations from the Red Sea and P. radiata from the Mediterranean Sea. Rev. Biol. Trop. 57 (3): 659-670. Epub 2009 September 30.
\end{abstract}

Key words: nacre shell, wild stocks, growth parameters, total mortality, Pteria sterna, South Baja California.

La concha nácar del Pacífico Americano Pteria sterna ha sido objeto de diversos estudios que describen su biología y ecología, logrando avances significativos en el desarrollo de técnicas eficientes de cultivo extensivo y producción de perlas cultivadas tipo "mabe" y perlas redondas, específicamente aplicadas en el área de la bahía de La Paz, B.C.S. y en la bahía de Bacochibampo, Sonora, México (Monteforte 1990, 1991, Cáceres-Martínez y Chávez-Villalba 1997, McLaurin et al. 1999, Nava et al. 2000).

La estimación de los valores de crecimiento y mortalidad de $P$. sterna constituyen parámetros de indudable importancia en la construcción de futuros modelos de dinámica poblacional base para el diseño de estrategias adecuadas de manejo, protección y perlicultivo. Los antecedentes de estudios sobre estos tópicos en $P$. sterna son ampliamente abordados en condiciones de cultivo extensivo (CáceresMartínez et al. 1991, 1992, Buckle-Ramírez et al. 1992, Del Río Portilla et al. 1992, Gaytan-Mondragón et al. 1992, Monteforte y García-Gasca 1994, Rangel-Dávalos y Cáceres 1994, Wright y Monteforte 1995, Aldana y Monteforte 1995, Monteforte et al. 1995, Wright 1997, MacLaurin et al. 1999, Young 1999, Monteforte et al. 2005).

P. sterna es considerada por el Acuerdo del Poder Ejecutivo Federal en la categoría de 
protección especial desde 1994 permitiendo únicamente la recolecta de semilla natural para fines de cultivo y experimentales (CariñoOlvera y Monteforte 1999). Actualmente han informado que existen actividades pesqueras de la concha nácar en las costas de Sonora, y las estadísticas de pesca gubernamentales la refieren con el nombre de callo de árbol. Para las costas de Baja California Sur no existen registros sobre esta actividad y la existencia y ubicación de los bancos naturales no han sido objeto de estudio. Por esta razón son escasas y se tiene pocas referencias de los valores de parámetros de crecimiento $\mathrm{y}$ mortalidad de esta especie en los bancos naturales, siendo de mayor relevancia los estudios de crecimiento de P. sterna en condiciones de repoblamiento en la bahía de La Paz (Saucedo-Lastra 1991, 1995, Saucedo y Monteforte 1997) quienes han obtenido estimaciones de parámetros de crecimiento.

Considerando la relevancia económica que guarda en la actualidad la concha nácar $P$. sterna, el conocimiento y estudio de las poblaciones naturales debe ser un objetivo prioritario para su manejo y protección. El presente estudio pretende estimar los valores de crecimiento y mortalidad de $P$. sterna en los bancos naturales del litoral oriental de Baja California Sur.

\section{MATERIALES Y MÉTODOS}

Sitios de estudio: El área de estudio abarcó una red de muestreo del litoral oriental del estado de Baja California Sur. El método utilizado para la búsqueda de los bancos naturales de $P$. sterna comprendió la exhaustiva prospección visual con empleo de buceo libre y SCUBA, de la bahía de La Paz, la bahía de Loreto, estero de Mulegé y las costas de Santa Rosalía. Se incluyó en esta búsqueda a la isla Ballena en el archipiélago de Espíritu Santo y la isla San José. En la bahía de Loreto la prospección comprendió una red de estaciones abarcando localidades en la costa de la bahía y las islas Coronados, Carmen y Danzante, así como el área de Ligüí e isla Montserrat, abarcando en total 38 estaciones en 2 bahías y 6 complejos insulares. El área total de búsqueda fue de $10000 \mathrm{~m}^{2}$ por año en el periodo de 1997-99. Los individuos silvestres de la concha nácar $P$. sterna fueron ubicados únicamente en cuatro sitios del litoral oriental de Baja California Sur: la costa de Santa Rosalía en las inmediaciones del estero San Luciano (estaciones: 1. El Farito y 2. El Barquito) en la desembocadura del estero de Mulegé (estación 3) y en el extremo sur de la isla Coronados (estación 4) en la bahía de Loreto (Fig. 1).

Estimación de la densidad en los bancos naturales: Se determinó la densidad poblacional a partir de la aplicación del método de cuadrantes. Para tal efecto se lanzó un cuadrante de $50 \mathrm{~m}$ de longitud y $2 \mathrm{~m}$ de observación a cada lado, correspondiendo a $250 \mathrm{~m}^{2}$ de observación, contando todos los individuos contenidos en el cuadrante. Para el caso de los bancos encontrados en Santa Rosalía la representación correspondió a un total de 5 cuadrantes (1 $250 \mathrm{~m}^{2}$ de prospección), para mayo de 1998. Para los bancos del estero de Mulegé e isla Coronados únicamente se registraron biometrías (medición dorsoventral MDV) en el sitio sin remoción de ostras de los bancos naturales.

Obtención de muestras: A partir de la determinación de la densidad se procedió a tramitar un permiso de pesca de fomento e investigación ante la oficialía de partes del departamento de pesquerías de la Delegación Federal de Pesca de B.C.S. (SEMARNAPBCS-04-1310/98), con una extracción de 30 ostras por mes para el estudio de la ecología y dinámica poblacional de concha nácar $P$. sterna en el área de Santa Rosalía, B.C.S. Las autoridades así determinaron que el tamaño de muestra no afectaría significativamente a los bancos naturales.

Con ayuda de pescadores de Santa Rosalía obtuvimos muestras mensuales de P. sterna de los bancos silvestres durante el periodo de 1997-99 ( $\mathrm{n}=233$ ostras) siendo recolectadas al azar mediante buceo con Hooka o compresor en las inmediaciones del estero San Luciano. El método al azar implica que en un área donde 
se establece un banco las ostras se recolectaron sin la aplicación de ningún criterio a priori de selección. Los pescadores recolectaron los racimos tal como eran encontrados siempre y cuando contuvieran el tamaño mínimo de muestra ya establecido, las ostras restantes eran devueltas al mar.

El número de ostras correspondió a un tamaño mínimo de muestra entre 23 y 30 ostras por mes provenientes de las estaciones de El Barquito y El Farito en las costas de Santa Rosalía. Conservamos las muestras en una solución de formaldehído al $10 \%$ en agua de mar en cubetas. Posteriormente fueron llevadas al laboratorio para el registro de la altura y peso. Las ostras se limpiaron de organismos epibiontes y del exceso de humedad con papel secante. El peso total de cada ostra se determinó en una balanza con precisión de $0.001 \mathrm{~g}$.

Con buceo libre exploramos las áreas rocosas y ubicamos de manera visual los sitios donde están emplazados bancos naturales de $P$. sterna comprendidos en la franja costera del estero de Mulegé y de la bahía de Loreto. Estos sitios mostraban el substrato adecuado para la proliferación de la concha nácar, principalmente donde eran abundantes los corales blandos de la especie Muricea californica, ya que los individuos de concha nácar estaban sujetos a esta especie de coral.

Durante la ubicación de los bancos naturales de $P$. sterna, registramos datos de altura de la concha sin extracción de organismos. Las ostras fueron medidas in situ con buceo SCUBA, para ello utilizamos un vernier de $0.01 \mathrm{~mm}$ de precisión. Registramos la longitud o medida dorso-ventral, distancia comprendida entre la charnela y el margen de las valvas excluyendo procesos de crecimiento.

Las ostras extraídas de los bancos de Santa Rosalía comprendieron 233 ostras de las cuales se obtuvieron biometrías en magnitud y peso. Con la prospección de bancos del estero de Mulegé y bahía de Loreto, sin extracción de ostras, reunimos 120 mediciones extra, lo que permitió reunir un total de 353 mediciones correspondientes a las magnitudes por individuo (altura) y con las cuales conformamos los histogramas de frecuencias de alturas para la estimación de parámetros de crecimiento. Con estas muestras reunimos 35.5 datos por "mes artificial" provenientes de diferentes bancos. Se ha demostrado que la formación de años virtuales son válidos para la determinación de los parámetros de crecimiento y que son representativos para el crecimiento de la especie, estos datos incluso han sido utilizados con fines de evaluaciones y predicciones pesqueras (Langi 1990, Guanco 1991).

Análisis de crecimiento y mortalidad: $\mathrm{Al}$ construir las distribuciones de frecuencias de alturas conjuntamos los datos de diferentes años para un mismo mes formando así un único "año artificial" o "año virtual", de esta forma aseguramos que la cantidad de datos de altura en cada histograma de frecuencias mensual cumple con la información mínima necesaria, según el teorema del límite central, para definir con claridad los componentes gaussianos de estas distribuciones. Diversos estudios han demostrado que la formación de "años artificiales" conjuntando los datos mensuales de tallas de diferentes años, permite calcular los parámetros de crecimiento mediante desplazamiento modal de los histogramas de frecuencias, y que es un método de cálculo confiable y predictivo de la variación del crecimiento. Alternativamente representa una metodología viable cuando se cuenta con escasa información de poblaciones muy dispersas, como en el caso de las ostras perleras del Golfo de California y que logísticamente es difícil para obtener una gran cantidad de datos en cortos periodos de tiempo. Le eficiencia de esta metodología queda demostrada en los estudios de crecimiento de Langi (1990) quien determinó los parámetros de crecimiento de tres especies de lutjánidos del archipiélago de Tonga, Pacífico Sur y los estudios de Guanco (1991) quien calculó los parámetros de crecimiento de la macarela Rastrelliger kanagurta en el mar de Visayas, Filipinas.

Pese a la recolecta de ostras del medio natural, y a la recopilación de datos de biometrías de ostras sin extracción, únicamente 
logramos reunir información de frecuencias de alturas correspondiente a diez meses. La estimación de los parámetros de crecimiento de von Bertalanffy para las alturas la realizamos usando el método ELEFAN I (Pauly y David 1981) contenido en el software FiSAT II (Gayanilo et al. 1995) y fue representada por la siguiente ecuación:

$$
L t=L_{\infty}\left[1-\exp \left(-K\left(t-t_{0}\right)+(C k / 2 \pi) \operatorname{sen} 2 \pi\left(t-t_{s}\right)\right)\right]
$$

Donde $L_{t}$ es la longitud a la edad $t, L_{\infty}$ es la altura asintótica, $K$ es la constante de crecimiento, $t_{0}$ es el tiempo en la cual la ostra inicia su crecimiento y $t_{s}$ es el periodo del año cuando el crecimiento es máximo.

Calculamos el valor máximo teórico de altura y su intervalo de confianza al $95 \%$ de valor de probabilidad. Utilizamos el método de Wetherall (1986) para estimar el intervalo de valores de $L_{\infty}$. Posteriormente los valores de $L_{\infty}$ y $K$ los depuramos mediante la rutina de búsqueda automática.

Estimamos el índice de desempeño del crecimiento $\left(\Phi^{\prime}\right)$ aplicando la ecuación derivada por Munro y Pauly (1983) en la forma de $\Phi$ ' $=\log _{10}(k)+2 \log _{10}\left(L_{\infty}\right)$, donde $L_{\infty}$ y $k$ son los parámetros de crecimiento.

El parámetro $\mathrm{t}_{0}$ definido como la "edad" hipotética que la ostra debiera tener para que su longitud fuese cero lo calculamos con la aproximación propuesta por Pauly (1979) según la ecuación $\log _{10}\left(-t_{0}\right)=-0.3922-0.2752$ $\log _{10} L_{\infty}-1.038 \log _{10} k$.

La máxima edad posible o longevidad la calculamos con la expresión $t_{\max }=(2.9957 / \mathrm{k})$ $+t_{0}$ considerando que la mayor talla observada en la naturaleza corresponde aproximadamente al $95 \%$ de $L_{\infty}$ según Taylor (1962) y Beverton (1963).

Estimamos la relación morfométrica entre la altura de la concha (medida dorsoventral, MDV) y el peso total de acuerdo a la ecuación derivada por Le Cren (1951), $\mathrm{W}_{(\mathrm{i})}=\mathrm{a} * \mathrm{~L}_{(\mathrm{i})}{ }^{\mathrm{b}}$ en donde $\mathrm{W}_{(\mathrm{i})}$ es el peso total en gramos, $\mathrm{L}_{(\mathrm{i})}$ es la altura (MDV) en milímetros, a y b son constantes.

La tasa instantánea de mortalidad total, Z, la determinamos mediante la curva de captura basada en tallas (Pauly 1983), considerando que no existe explotación de los bancos naturales correspondería a la tasa instantánea de mortalidad natural.

\section{RESULTADOS}

Densidad: Las estimaciones de densidad durante agosto de 1997 mostraron que en los sitios de muestreo en las costas de Santa Rosalía existen 60 ostras $/ 250 \mathrm{~m}^{2}$ lo que corresponde a 24 individuos de concha nácar por cada $100 \mathrm{~m}^{2}$ de observación en las estaciones El Barquito y El Farito (Fig. 1). Las muestras fueron recolectadas por los buzos de la cooperativa de Santa Rosalía a una profundidad entre 30 y $50 \mathrm{~m}$, el banco se extiende hacia el norte a la altura de la comunidad de Santa Gertrudis e incluso es posible que más hacia el norte. Esta información fue evaluada por la oficialía de partes del departamento de pesquerías de la delegación federal del estado de Baja California Sur para el trámite del permiso de extracción de ostras para fines de investigación. Las estaciones del estero de Mulegé e isla Coronados mostraron muy pocas ostras (6 ostras / $250 \mathrm{~m}^{2} ; 2.4$ ostras $/ 100 \mathrm{~m}^{2}$ ) en el cuadrante de evaluación, por lo que no se extrajeron ostras de estos sitios.

Parámetros de crecimiento: Los datos de frecuencias de altura comprendieron la medición de 353 ostras de los bancos silvestres de la costa oriental de Baja California Sur. Aplicando el método de Wetherall (1986) determinamos que el intervalo de la altura máxima asintótica fue de 148.38 a $156.08 \mathrm{~mm}$, posteriormente procedimos a la búsqueda de los valores de $L_{\infty}$ dentro de este intervalo y de $k$ mediante la rutina de búsqueda automática del ELEFAN I. La determinación del valor apropiado de $k$ con un valor fijo de $L_{\infty}$ la efectuamos utilizando el valor más alto de la función Rn como criterio, permitiendo en este proceso variación estacional. Los valores de parámetros de crecimiento de la curva de von Bertalanffy (Fig. 2) fueron: $L_{\infty}=156.02 \mathrm{~mm}, k=0.48 \mathrm{año}^{-1}, t_{0}=-0.216$ años, $\mathrm{C}=0.43, \mathrm{WP}=0.73$ y $t_{s}=0.5(\mathrm{Rn}=$ 


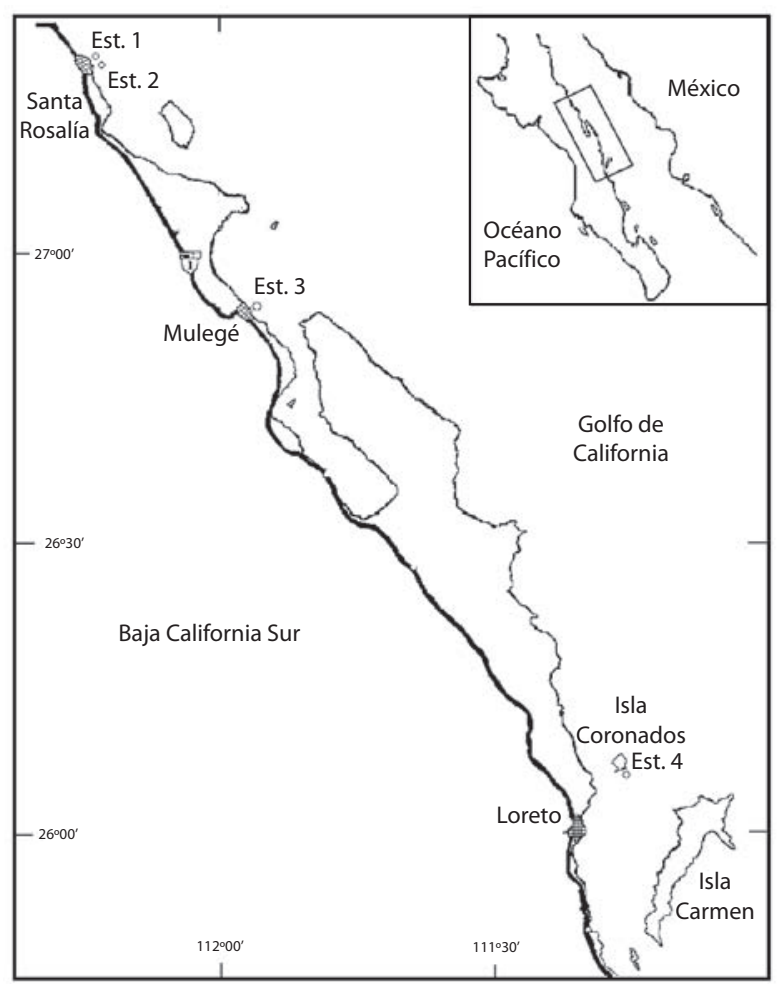

Fig. 1. Localidades de muestreo: Santa Rosalía (Est.1: El Farito, Est. 2: El Barquito), Mulegé (Est. 3: Estero de Mulegé) y bahía de Loreto (Est. 4: Coronados).

Fig. 1. Sampling sites: Santa Rosalía (Est. 1: El Farito, Est. 2: El Barquito), Mulegé (Est. 3: Estero de Mulegé) and bahía de Loreto (Est. 4: Coronados).

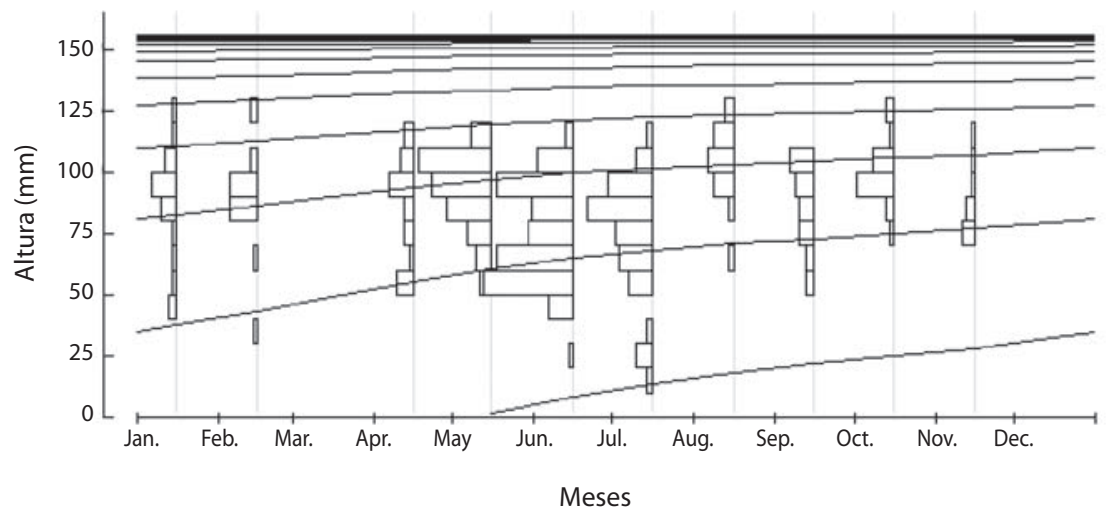

Fig. 2. Estimación del crecimiento de P. sterna con el análisis de progresión modal en un año virtual. Valor de bondad de ajuste $(\mathrm{Rn}=0.267)$.

Fig. 2. Growth estimation of $P$. sterna with the analysis of modal progression in one virtual year. Value of the goodness-offit $(\mathrm{Rn}=0.267)$. 
0.267), donde C representa la intensidad de la oscilación estacional y WP la época del año donde ocurre el mayor retardo en crecimiento. Saucedo y Moteforte (1997) estimaron valores de los parámetros de crecimiento por progresión modal para $P$. sterna en condiciones de repoblamiento obteniendo una $L_{\infty}=100$ $\mathrm{mm}, k=0.69 \mathrm{año}^{-1}, \mathrm{C}=0.3$ y WP $=0.5$; también calcularon estos parámetros aplicando los métodos de regresión y mediante procesos de iteración basados en el algoritmo de Marquardt (Cuadro 1).

Considerando que el parámetro de crecimiento $k$ está vinculado a la longevidad de las ostras más viejas, y que representa el $95 \%$ de la talla máxima observada en la naturaleza, calculamos que para $P$. sterna de bancos naturales, con base en el valor de $k=0.48$ año $^{-1} \mathrm{y}$ $L_{t}=122.8 \mathrm{~mm}$ (longitud máxima registrada en las muestras), el valor de longevidad para la especie es de 3.0064 años.

Índice de desempeño del crecimiento: El índice de desempeño del crecimiento $\Phi$ ' puede ser igual en la misma especie o entre taxones estrechamente relacionados y entre diferentes bancos de la misma especie (Moreau et al. 1986). El valor de $\Phi$ ' para $P$. sterna de bancos naturales en el litoral oriental de Baja California Sur de acuerdo a la fórmula de Munro y Pauly (1983) es igual a $\Phi^{\prime}=4.068$. El Cuadro 2 muestra los diferentes valores de $\Phi$ ' para individuos de P. sterna comparando el crecimiento en condiciones de repoblamiento con respecto a la estimación obtenida para concha nácar en bancos naturales. La $\Phi^{\prime}$ de $P$. sterna en el litoral oriental de Baja California Sur fue mayor a las obtenidas para la misma especie

\section{CUADRO 1}

Parámetros de crecimiento de Pteria sterna en condiciones de repoblamiento calculados por diferentes métodos en comparación con las de ostras de bancos silvestres del litoral de Baja California Sur

TABLE 1

Growth parameters of Pteria sterna, under repopulation conditions, calculated by different methods in comparison to the conditions for oysters in wild banks of the coastline in Southern Baja California

$\begin{array}{lccccc}\text { Autor y método de estimación } & L_{\infty} & k & t_{0} & \text { C } & \text { WP } \\ \text { Saucedo y Monteforte (1997) ELEFAN I } & 100 & 0.69 & ---- & 0.3 & 0.5 \\ \text { Saucedo y Monteforte (1997) Regresión } & 67.363 & 0.1448 & -6.445 & ----- & ---- \\ \text { Saucedo y Monteforte (1997), Iteración. } & 68.822 & 0.1224 & -7.173 & ---- & ---- \\ \text { Wright } \text { et al. } \text { (presente estudio), ELEFAN I } & 156.02 & 0.48 & -0.216 & 0.43 & 0.73\end{array}$

\section{CUADRO 2}

Índice de desempeño de crecimiento $\Phi^{\prime}$ para individuos de $\mathrm{P}$. sterna en condiciones de repoblamiento comparados con el de ostras de bancos silvestres

TABLE 2

Growth performance index $\Phi$ 'for individuals of $\mathrm{P}$. sterna under repopulation conditions in comparison to the ones for oysters in wild banks

\begin{tabular}{|c|c|c|c|}
\hline Autor & Localidad & Método de cálculo de $L_{\infty}$ y $k$ & $\Phi^{\prime}$ \\
\hline Saucedo y Monteforte (1997) & Bahía de la Paz, & ELEFAN I & 3.839 \\
\hline Saucedo y Monteforte (1997) & Bahía de la Paz & Regresión & 2.818 \\
\hline Saucedo y Monteforte (1997) & Bahía de la Paz & Iteración & 2.763 \\
\hline $\begin{array}{l}\text { Wright et al. } \\
\text { (presente estudio) }\end{array}$ & $\begin{array}{l}\text { Litoral oriental de Baja } \\
\text { California Sur }\end{array}$ & ELEFAN I & 4.068 \\
\hline
\end{tabular}




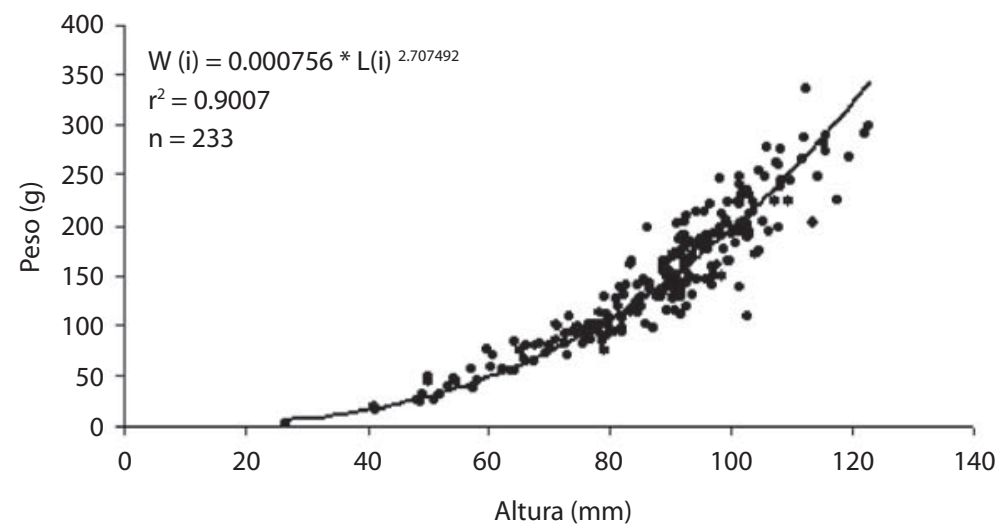

Fig. 3. Relación peso-altura de la concha nácar P. sterna en el litoral oriental de Baja California Sur.

Fig. 3. Weight-height relation of the nacre shell P. sterna in the oriental coastline of Southern Baja California.

en condiciones de repoblamiento. En opinión de Saucedo y Monteforte (1997) los valores de los parámetros de crecimiento se vieron afectados por las condiciones inherentes al experimento y las tallas máximas alcanzadas en el ensayo de repoblamiento. En nuestro estudio se muestra que la prospección de los bancos naturales de concha nácar del litoral oriental de Baja California Sur logró obtener la representación de un mayor espectro de tallas, incluyendo las ostras más grandes encontradas en la naturaleza, logrando con ello un ajuste con mayor precisión de la ecuación de crecimiento de la especie.

Relación peso-altura: La relación pesoaltura es requerida en análisis pesqueros para estimar la dinámica y determinar el tamaño de la población, ya que la tasa de incremento en peso refleja la influencia de los factores ecológicos de un hábitat y cómo estos afectan a los organismos (Mohammed y Yassien 2003). La Fig. 3 muestra la relación entre la altura de la concha y el peso de P. sterna provenientes de Santa Rosalía, la fórmula resultante es: $\mathrm{W}_{(\mathrm{i})}$ $=0.000756 * \mathrm{~L}_{(\mathrm{i})} 2.707492$ con un coeficiente de determinación de $\mathrm{r}^{2}=0.9007, \mathrm{y} \mathrm{n}=233$ ostras.

Esta relación fue isométrica con un valor de la pendiente $b=2.7075$ y no fue significativamente diferente del valor isométrico, $\mathrm{p}<0.1$.
Mortalidad total: En la curva de captura convertida a longitud, Fig. 4, la pendiente de la línea ajustada fue igual a $\mathrm{Z}=3.34 \mathrm{anno}^{-1}$, siendo la ecuación de regresión: $\operatorname{Ln}(\mathrm{N} / \Delta \mathrm{t})=11.389$ $3.337 \mathrm{t}\left(\mathrm{r}^{2}=0.99\right)$. Es importante denotar que actualmente no existen antecedentes de estimaciones de mortalidad para ostras de bancos

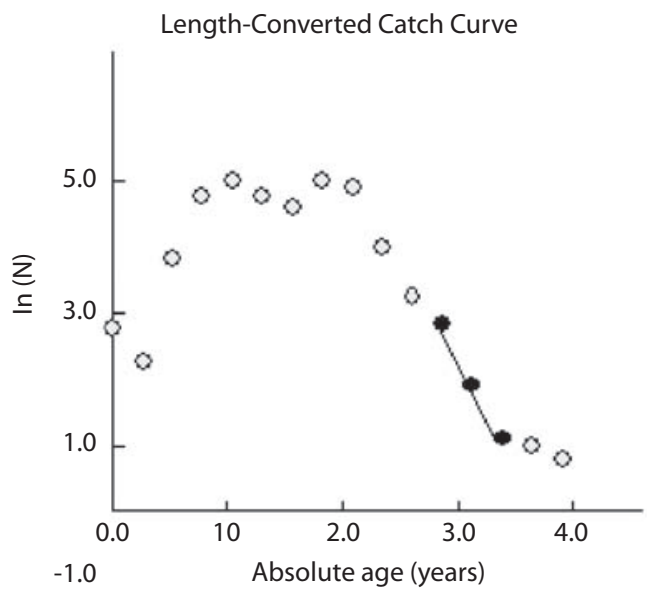

Fig. 4. Curva de captura convertida a longitud para estimar el coeficiente de mortalidad total (Z) de P. sterna.

Fig. 4. Lenght converted catch curve to estimate the total mortality coefficient (Z) of P. sterna. 
naturales del género Pteria, no obstante, constituyen especies de singular importancia y base de la perlicultura en diferentes localidades en el mundo, como lo son P. sterna en la bahía de Bacochibampo, Sonora, México; P. penguin y $P$. albina en Asia y Australia, así como en los estudios que actualmente se están desarrollando sobre la potencialidad del cultivo de $P$. colymbus en Venezuela y Colombia.

Sin embargo contamos con algunas referencias sobre estimaciones de mortalidad para ostras perleras de bancos naturales para especies del género Pinctada. Yassien (1998) estimó que $\mathrm{Z}=2.47$ año $^{-1}$ para Pinctada radiata (= fucata) recolectada en el Mar Rojo. Para el caso de individuos del género Pinctada se considera que en términos generales las condiciones ambientales como los regímenes de temperatura y las variaciones geográficas de productividad determinan significativamente el crecimiento y la mortalidad de ostras en cultivo extensivo y en los bancos naturales (Pouvreau y Prasil 2001). Alagarswami y Chellam (1976) comprobaron que los problemas de fouling y organismos horadadores son probables causas de mortalidad de Pinctada fucata en el Golfo de Mannar para ostras de diferentes edades.

\section{DISCUSIÓN}

La concha nácar $P$. sterna en el litoral oriental de Baja California Sur mostró los siguientes parámetros de crecimiento: $L_{\infty}=$ $156.02 \mathrm{~mm}, k=0.48$ año $^{-1}, t_{0}=-0.216$ años, $\mathrm{C}=0.43$ y WP $=0.73$. La comparación de los valores calculados en el presente estudio con los obtenidos en condiciones de repoblamiento denota que no existe un patrón definido para estos valores, mostrándose una diferencia con respecto al crecimiento de concha nácar de los bancos naturales. Los diferentes métodos utilizados para la estimación de los parámetros de crecimiento pueden ser un factor para la diferencia en las estimaciones, también es de suma importancia considerar las diferencias en condiciones ecológicas entre localidades. La ausencia de tallas grandes en las muestras afecta la estimación de crecimiento mediante el método de ELEFAN I resultando en valores elevados del coeficiente de crecimiento $\mathrm{k}$, tal parece ser el caso de aquellos reportados por Saucedo y Monteforte (1997). Es de relevancia anotar que todos los métodos para estimar $L_{\infty}$ a partir de frecuencia de tallas son muy sensibles a las tallas máximas presentes en la muestra.

Estimamos un valor de índice de desempeño del crecimiento de $\Phi^{\prime}=4.068$. El valor de $\Phi^{\prime}$ de $P$. sterna fue mayor para las ostras de bancos naturales en comparación con los valores obtenidos para ostras en condiciones de repoblamiento en la bahía de La Paz, lo anterior puede esperarse considerando las condiciones de este experimento donde los individuos de $P$. sterna fueron colocados en células de repoblamiento con una edad inicial de 1.5 años y $42.8 \mathrm{~mm}$ de altura. En este sentido las muestras de frecuencias de tallas de concha nácar de los bancos naturales del litoral oriental de Baja California Sur comprendieron un mayor espectro de tallas en la muestra, considerando desde las tallas de las semillas hasta las mayores tallas de ostras encontradas en la naturaleza. El muestreo al azar de los bancos de Santa Rosalía incluyó ostras de mayor altura que las observadas en el experimento de repoblamiento, así obtuvimos menor sesgo en el ajuste de la curva de von Bertalanffy, y por consiguiente logramos el mejor ajuste posible del modelo.

Los parámetros de crecimiento de $P$. ster$n a$ en condiciones de repoblamiento fueron afectados por las condiciones mismas del experimento, así los parámetros de crecimiento fueron diferentes a los estimados en el presente estudio para individuos de concha nácar en los bancos naturales. Saucedo-Lastra (1995) considera que los factores ambientales característicos del repoblamiento, tales como el tipo de substrato manejado que fue rajuela, un tipo de roca basáltica de la región, la cantidad de luz recibida, la disponibilidad de alimento y principalmente la densidad poblacional a la que fueron mantenidos los organismos dentro de las canastas de repoblamiento, influenciaron seguramente la tasa de crecimiento y la talla que alcanzaron los mismos al término del experimento. 
Es conocido que son diversas las características bióticas y abióticas que regulan el crecimiento (en talla o peso) de los bivalvos, como las variaciones estacionales de la temperatura del agua, luz y alimento, el ciclo reproductivo de los organismos, los patrones de almacenamiento y la utilización de la energía disponible (Gabbot 1976, Seed 1980, Barber y Blake 1981, en Saucedo y Monteforte 1997).

El presente estudio fue posible gracias al posicionamiento de los bancos silvestres de $P$. sterna. Los resultados de la exploración muestra su ubicación en las localidades de Santa Rosalía, estero de Mulegé e isla Coronados en la bahía de Loreto, considerando este último como el límite sur de la distribución de los bancos naturales de la especie para el litoral oriental de Baja California Sur. Así denotamos que existen factores bióticos y abióticos que determinan la disminución de la abundancia de P. sterna hacia la bahía de La Paz. Considerando que en esta bahía se recolecta semilla y se cultiva concha nácar de manera extensiva (Cáceres-Martínez et al. 1992, Monteforte y García-Gasca 1994, Wright y Monteforte 1995), es muy probable que un factor biótico, como la presencia y distribución de especies de depredadores con afinidad tropical, determinen este patrón de distribución, y que la mortalidad de individuos de bancos naturales en estas áreas geográficas sea determinada por eventos depredatorios (Saucedo y Monteforte 1997). Por otra parte el crecimiento esta influenciado por factores ambientales como los regímenes de temperatura y variaciones en la productividad primaria del área, los cuales se ha demostrado que son factores de particular importancia que afectan a ostras de la familia Pteriidae en otros sitios como la Polinesia Francesa (Pouvreau y Prasil 2001).

Los bancos de P. sterna de mayor densidad fueron ubicados en la desembocadura del estero San Luciano en Santa Rosalía, y en el estero de Mulegé, donde es evidente un aporte importante de materia orgánica de origen terrígeno, mostrando la relevancia de este factor en el emplazamiento de los bancos naturales y probablemente influenciando de manera importante el crecimiento y mortalidad de las ostras en estas poblaciones. En ambos casos, P. sterna se encontró asociada a colonias de Gorgonacea en el área ecotonal entre el ambiente marino, y donde hay influencia de aportes terrígenos a las afueras de los esteros. No obstante, en el caso de la estación 4 Coronados, el ambiente es únicamente de influencia marina y P. sterna también estuvo asociada a individuos de Gorgonacea.

Los altos valores de $\mathrm{Z}=3.34 \mathrm{año}^{-1}$ para $P$. sterna en el litoral oriental de Baja California Sur muestran un acelerado proceso de mortalidad en las tallas intermedias como observamos en la curva de captura convertida a longitud. En este sentido las referencias de estimaciones de mortalidad en Pteriidae son más diversas para el género Pinctada. Yassien (1998) estimó un valor de $\mathrm{Z}=2.47$ año $^{-1}$ para $P$. radiata $(=$ fucata) en el Mar Rojo. Yassien et al. (2000) estimaron un valor de $\mathrm{Z} / \mathrm{k}=5.415$ y un $\mathrm{k}=$ $0.56\left(\mathrm{Z}=3.0324\right.$ año $\left.^{-1}\right)$ para $P$. radiata en el este del mar Mediterráneo, mientras que para la misma especie en aguas de Qatar, la línea recta de regresión de curva de captura convertida a longitud fue ajustada a los puntos de longitud 60.0 a $125.0 \mathrm{~mm}$ de la gráfica, así la pendiente de la línea ajustada fue igual a $\mathrm{Z}=$ $1.74 \mathrm{año}^{-1}$ (Mohammed y Yassien 2003). Lo anterior sugiere que en términos generales se ha obtenido altos valores de mortalidad total para especies de la misma familia en diferentes zonas geográficas considerando la metodología de la curva de captura convertida a longitud. La importancia de los diferentes factores ambientales en determinar el grado de mortalidad en poblaciones de Pteriidae ha sido estudiada previamente para individuos del género Pinctada (Pouvreau y Prasil 2001), sin embargo han sido pobremente estudiadas para ostras perleras del género Pteria.

\section{AGRADECIMIENTOS}

Agradecemos al Consejo Nacional de Ciencia y Tecnología (CONACYT) por el apoyo económico para la realización del presente estudio y por concederme la beca para 
estudios de doctorado en ciencias marinas. También agradecemos al Fondo Mexicano para la Conservación de la Naturaleza (FMCN) por los fondos para el proyecto "Estudio de las comunidades marinas litorales de la bahía de Loreto e islas circunvecinas".

\section{RESUMEN}

La concha nácar Pteria sterna sustenta los perlicultivos en México, y es la base de producción de perlas en el continente Americano. Está bajo protección especial desde 1994, y solo puede capturarse semilla natural para cultivo e investigación. Determinamos su crecimiento y mortalidad en bancos silvestres, registrando la altura de ostras de Santa Rosalía, Mulegé y Bahía de Loreto (1997-99). La longitud máxima estimada fue $130.35 \mathrm{~mm}(124.22-136.48 \mathrm{~mm}$, $P>0.95)$. Las frecuencias de longitudes se ordenaron en un "año virtual" (Guanco 1991). Los parámetros de crecimiento de von Bertalanffy estimados con el programa ELEFAN I fueron: $L_{\infty}=156.02 \mathrm{~mm}, k=0.48 \mathrm{anno}^{-1}, t_{0}=-0.216$ años, $\mathrm{C}=$ 0.43 y WP $=0.73$; el índice de desempeño de crecimiento fue $\Phi^{\prime}=4.068$. La mortalidad total fue de $Z=3.34$ año ${ }^{-1}$ por longitud convertida a curva de captura. La regresión del peso total y la altura tuvo la expresión $\mathrm{W}=0.000756$ * $\mathrm{L}_{\text {(i) }} 2.7075\left(\mathrm{r}^{2}=0.90, \mathrm{n}=233\right)$. Mostramos la diferencia entre los parámetros de crecimiento en condiciones de repoblamiento experimental y aquellos de individuos silvestres de $P$. sterna. El valor de mortalidad total es comparable a aquellos de Pinctada fucata en el mar Rojo o P. radiata en el mar Mediterráneo.

Palabras clave: concha nácar, bancos naturales, parámetros de crecimiento, mortalidad total, Pteria sterna, Baja California Sur.

\section{REFERENCIAS}

Alagarswami, K. \& A. Chellam. 1976. On fouling and boring organisms and mortality of pearl oysters in the farm at Veppalodai, Gulf of Mannar. Indian J. Fish. 23: $10-22$.

Aldana, C. \& M. Monteforte. 1995. Spat collection, growth and survival of pearl oyster Pteria sterna under extensive culture conditions in bahía de La Paz, South Baja California, Mexico. Resumen 5. In Book of Abstracts. Aquaculture '95, February 1-4, San Diego, California. World Aqua. Soc.

Barber, B.J. \& N.J., Blake. 1981. Growth and reproduction of the bay scallop, Argopecten irradians (Lamark) at its southern distributional limit. J. Exp. Mar. Biol. Ecol. 52: 121-134.
Beverton, R.J.H. 1963. Maturation, growth and mortality of clupeid and engraulid stocks in relation to fishing. Rapp. P.-V. Réun. CIEM. 154: 44-67.

Buckle-Ramírez, L.F., D. Voltolina-Lobina, E. MoralesGuerrero \& F. Valenzuela-Buriel. 1992. Spat settlement and growth of Pteria sterna (Gould) (Mollusca, Bivalvia) in bahía de Los Angeles, Baja California, México. Trop. Ecol. 33: 137-147.

Cáceres-Martínez, C., J. Cortés-Salazar \& J. ChávezVillalba. 1991. Reclutamiento de jóvenes de moluscos bivalvos en Baja California Sur, México, p. 151-165. In S. Gúzman del Proo (ed.). Taller México-Australia sobre Reclutamiento de Recursos Bentónicos de Baja California, 25-29 de Noviembre 1991, La Paz, B.C.S., México. SEPESCA-IPN.

Cáceres-Martínez, C., C.A. Ruiz-Verdugo \& D.H. RamírezFilipini. 1992. Experimental collection of pearl oyster Pinctada mazatlanica and Pteria sterna, on a filament substrate. J. World Aquac. Soc. 23: 232-239.

Cáceres-Martínez, C. \& J. Chavez-Villalba. 1997. Pearl oyster culture in Baja California Sur, Mexico. J. World Aqua. Soc. 33-38.

Cariño-Olvera, M.M. \& C. Cáceres-Martínez. 1990. La Pesquería de perlas y la perlicultura en Sudcalifornia a principios de siglo. Ser. Cient. UABCS (La Paz), México. 1: 1-6.

Cariño-Olvera, M.M. \& M. Monteforte. 1999. El primer emporio perlero sustentable del mundo. UABCS CONACULTA-FONCA, México. 325 p.

Del-Rio-Portilla, M.A., A.D. De-Araujo \& D. Voltolina. 1992. Growth of the pearl oyster Pteria sterna under different thermic and feeding conditions. Mar. Ecol. Prog. Ser. 89: 221-227.

Gabbot, P.A. 1976. Energy metabolism, p. 293-355. In B.L Bayne (ed.). Marine Mussels. Cambridge University Press, London and New York.

Gayanilo, F.C. Jr., P. Sparre \& D. Pauly. 1995. The FiSAT users guide. FAO computerized information series fisheries. 99, ICLARM, DIFMAR, Rome. 265 p.

Gaytan-Mondragón, I., C. Cáceres-Martínez \& M. TobiasSánchez. 1992. Growth of the pearl oysters Pinctada mazatlanica and Pteria sterna in different culture structures at La Paz Bay, Baja California Sur, Mexico. J. World Aqua. Soc. 24: 541-546.

Guanco, M.R. 1991. Growth and mortality of indian mackerel Rastrelliger kanagurta (Scombridae) in the Visayas Sea, Central Phillippines. Fishbyte. ICLARM, Manila Phillippines. 9: 13-15. 
Langi, S. 1990. The applicability of ELEFAN for use in analyzing three species of deepwater snappers in Tonga (Etelis coruscans, Pristipomoides flavipinnis y $P$. filamentosus, Fam. Lutjanidae). Fishbyte. ICLARM, Manila Phillippines. 8: 21-25.

Le Cren, D.E. 1951. The length weight relationship and seasonal cycle in gonad weight and condition in the perch Perca fluviatilis. J. Anim. Ecol. 20: 201-219.

McLaurin, D., E. Arizmendi, S. Farell \& M. Nava. 1999. Pearls and pearl oysters from the Gulf of California, México, an update. Aust. Gemm. 20: 239-245.

Mohammed, S.Z. \& M.H. Yassien. 2003. Population parameters of the pearl oyster Pinctada radiata (Leach) in Qatari Waters, Arabian Gulf. Turk. J. Zool. 27: $339-343$

Monteforte, M. 1990. Ostras perleras y perlicultura: situación actual en los principales países productores y perspectivas para México. Ser. Cient. UABCS. 1: 13-18.

Monteforte, M. 1991. Las perlas, leyenda y realidad: un proyecto actual de investigación científica. Revista Panorama. Nueva Época. UABCS. 38: 28-31.

Monteforte, M. \& A. García-Gasca. 1994. Spat collection studies on pearl oysters Pinctada mazatlanica and Pteria sterna (Bivalvia: Pteriidae) in bahía de La Paz, South Baja California, México. Hydrobiologia 291: 21-34.

Monteforte, M., E. Kappelman-Piña \& B. López-Espinosa 1995. Spatfall of pearl oyster, Pteria sterna (Gould), on experimental collectors at bahía de La Paz, South Baja California, Mexico. Aqua. Res. 26: 497-511.

Monteforte, M., H. Bervera, J.J. Ramírez, P. Saucedo \& C.O. López. 2005. Effect of stocking density on growth and survival of the rainbow pearl oyster Pteria sterna (Gould 1852) during nursery and late culture in bahía de La Paz, Baja California Sur, México. Aqua. Inter. 13: 391-407.

Moreau, J., C. Bambino \& D. Pauly. 1986. Indices of overall fish growth performance of 100 tilapia (Cichlidae) populations, p. 201-206. In J.L. Maclean, L.B. Dizon \& L.V. Hosillos (eds.). The first asian fisheries forum. Asian Fish. Soc. Manila, Philippines.

Munro, J.L. \& D. Pauly. 1983. A simple method for comparing growth of fishes and invertebrates. ICLARM. Fishbyte 1: 5-6.

Nava, M., E. Arizmendi, S. Farell \& D. Mclaurin. 2000. Evaluation of success in the seeding of round nuclei in Pteria sterna (Gould 1851), a new species in pearl culture. SPC Pearl Oyster Inf. Bull. 14: 12-16.

Pauly, D. 1979. Theory and management of tropical multispecies stocks: a review, with emphasis on the Southeast Asian demersal fisheries. ICLARM Stud. Rev. 1: 35.

Pauly, D. 1983. Some simple methods for the assessment of tropical fish stocks. FAO Fish. Tech. Pap. 234: 1-52.

Pauly, D. \& N. David. 1981. ELEFAN I, a BASIC program for the objective extraction of growth parameters from length-frequencies data. Meeresforsch. 28: 205 211.

Pouvreau, S. \& V. Prasil. 2001. Growth of the black-lip pearl oyster, Pinctada margaritifera, at nine culture sites of French Polynesia: synthesis of several sampling designs conducted between 1994 and 1999. Aquat. Living Resour. 14: 155-163.

Rangel-Dávalos, C. \& C. Cáceres-Martínez. 1994. Pearl oyster culture in México. In Abstracts of the International Pearl Conference and Exposition, Pearls '94. J. Shellfish Res. 13: 347-348.

Saucedo-Lastra, P.E. 1991. Ensayo sobre repoblamiento de bancos naturales de concha nácar Pteria sterna y madreperla Pinctada mazatlanica (Bivalvia: Pteriidae) en el Merito, Bahía de La Paz, Sudcalifornia, México. Tesis de Licenciatura, UNAM. 75 p.

Saucedo-Lastra, P.E. 1995. Crecimiento, relaciones alométricas y reproducción de las ostras perleras Pinctada mazatlanica y Pteria sterna (Bivalvia: Pteriidae) en condiciones de repoblamiento en el Merito, Bahía de La Paz, Baja California Sur, México. Tesis de Maestría en Ciencias, CICIMAR, México. 101 p.

Saucedo, P.E. \& M. Monteforte. 1997. In situ growth of pearl oysters Pinctada mazatlanica (Hanley 1856) and Pteria sterna (Gould 1851) under repopulation conditions at bahía de La Paz, Baja California Sur, México. Aqua. Res. 28: 367-378.

Seed, R. 1980. Shell growth and form in the Bivalvia. In D.C. Rhoads \& R.A. Lutz (eds.). Skeletal growth of aquatic organisms. Biological Records of Environmental Change. Plenum Press, New York.

Taylor, C.C. 1962. Growth equation with metabolic parameters. J. Cons. CIEM. 27: 270-86.

Wetherall, J.A. 1986. A new method for estimating growth and mortality parameters for length frequency data. ICLARM. Fishbyte 4: 12-14. 
Yassien, M.H. 1998. Biological and ecological studies on the pearl oyster, Pinctada radiata (Mollusca, Lamellibranchia) from the Red Sea, with special reference to its tolerance to water pollution. Ph.D Thesis, Faculty of Science, Ain Shams University, Cairo, Egypt. 191 p.

Yassien, M.H., F.A. Abdel-Razek \& R.W. Kilada. 2000. Growth estimates of the pearl oyster, Pinctada radia$t a$, from the Eastern Mediterranean. Egypt. J. Aquat. Biol. Fish. 4: 105-118.

Young, T. 1999. Exploring the potential of indigenous species for aquaculture in bahía Magdalena: Growth rate of cultivated concha nácar (Pteria sterna). Swarthmore College, Swarthmore, PA. School for
Field Studies, Centro de Estudios Costeros, Puerto San Carlos, B. C. S. 1-7.

Wright, H. \& M. Monteforte. 1995. Ecology of pearl oyster spat collection in bahía de La Paz, South Baja California, México: Temporal and vertical distribution, substrate selection, associated species. Resume 535. In Abstracts of International Congress Aquaculture '95, San Diego, California.

Wright, L. H. 1997. Ecología de la captación de semilla de madreperla Pinctada mazatlanica y concha nácar Pteria sterna (Bivalvia: Pteriidae), en la isla Gaviota, bahía de La Paz, B. C. S., México. Tesis de Maestría en Ciencias, CICIMAR-IPN, La Paz, B. C. S., México. 139 p. 